\title{
Acute Basilar Artery Occlusion: Outcome of Mechanical Thrombectomy with Solitaire Stent within 8 Hours of Stroke Onset
}

\author{
J.M. Baek, W. Yoon, S.K. Kim, M.Y. Jung, M.S. Park, J.T. Kim, and H.K. Kang
}

\begin{abstract}
BACKGROUND AND PURPOSE: Mechanical thrombectomy with a stent retriever applied shortly after symptom onset could increase good functional outcomes and improve survival in patients with acute basilar artery occlusion, but this has not yet been studied. This study evaluated the efficacy and safety of mechanical thrombectomy with a Solitaire stent within 8 hours of stroke onset in patients with acute basilar artery occlusion.
\end{abstract}

MATERIALS AND METHODS: We analyzed 25 consecutive patients with acute basilar artery occlusion who were treated with mechanical thrombectomy by use of the Solitaire stent within 8 hours of stroke symptom onset. Successful recanalization was defined as TICI grade $2 \mathrm{~b}$ or 3 . Good outcome was defined as mRS score of $0-2$ at 3 months. Clinical and radiologic data in patients with good outcomes were compared with those with poor outcomes.

RESULTS: Successful recanalization was achieved in 96\% (24/25) of patients, and 48\% (12/25) of patients had good outcomes. Eighty-eight percent $(22 / 25)$ of patients survived to 3 months. The median NIHSS score on admission was significantly lower in patients with good outcomes than in those with poor outcomes (9.5 versus $14, P=.005)$. Procedure-related complications occurred in 2 patients (8\%). No symptomatic intracerebral hemorrhages occurred.

CONCLUSIONS: The current study suggests that mechanical thrombectomy by use of a Solitaire stent within 8 hours of stroke onset increases good outcomes and improves survival in patients with acute basilar artery occlusion.

ABBREVIATIONS: BAO = basilar artery occlusion; IA = intra-arterial; BASICS = Basilar Artery International Cooperation Study

$A^{-}$ cute basilar artery occlusion (BAO) is associated with a very poor outcome and has the highest mortality rate among intracranial large-vessel occlusions. ${ }^{1,2}$ In a prospective, observational study (BASICS [Basilar Artery International Cooperation Study]), 27 patients were treated conservatively because the patients were either comatose $(n=26)$ or tetraplegic $(n=1)$ at the time of presentation; $96.3 \%$ (26/27) of these patients died, and the 1 remaining patient had an mRS score of 5 at 1 month. ${ }^{2}$

Clinical outcomes were unsatisfactory, even when patients with acute $\mathrm{BAO}$ were treated with intravenous or intra-arterial (IA) pharmacologic thrombolysis. A systematic analysis including 420 patients showed that the rates of death or dependency

Received July 24, 2013; accepted after revision October 16.

From the Departments of Radiology (J.M.B., W.Y., S.K.K., M.Y.J., H.K.K.) and Neurology (M.S.P., J.T.K.), Chonnam National University Medical School, Chonnam National University Hospital, Gwangju, Republic of Korea.

Please address correspondence to Woong Yoon, MD, Department of Radiology, Chonnam National University Hospital, 671 Jebong-Ro, Dong-gu, Gwangju, 501-757, Republic of Korea; e-mail: radyoon@jnu.ac.kr

http://dx.doi.org/10.3174/ajnr.A3813 were $78 \%(59 / 76)$ in patients treated with IV thrombolysis and $76 \%(260 / 344)$ in those treated with IA thrombolysis. ${ }^{3}$

In the past few years, the introduction of stent-type clot removal devices (stent retrievers) have initiated a new era in endovascular stroke therapy. Two randomized, controlled trials and many case series showed that stent retrievers can achieve a high rate of successful recanalization of up to $90 \%$, thus improving the clinical outcome in patients with acute intracranial large-vessel occlusion. ${ }^{4-11}$ Most studies were focused on anterior circulation stroke.

The efficacy of stent retrievers for treating acute BAO was reported in several recent case series. ${ }^{12-14}$ These previous studies used a longer time window of up to 24 hours for mechanical thrombectomy in patients with acute BAO. However, BASICS showed that early recanalization therapy in patients with acute $\mathrm{BAO}$ is associated with a more favorable outcome, and all patients with severe stroke treated $>9$ hours after symptom onset had poor functional outcome despite IV or IA thrombolysis. ${ }^{15}$ In this regard, the use of mechanical thrombectomy with a stent retriever within a shorter time period from symptom onset would increase 
the patient's chances for a good functional outcome and decrease the mortality rate in patients with acute $\mathrm{BAO}$, but this has not yet been studied. The aim of this study was to evaluate the efficacy and safety of mechanical thrombectomy with a stent retriever (Solitaire; Covidien, Irvine, California) as a first-line endovascular therapy within 8 hours of stroke onset in patients with acute BAO.

\section{MATERIALS AND METHODS}

\section{Patients}

Since December 2010, we prospectively collected data of patients with acute ischemic stroke treated with mechanical thrombectomy. The clinical and angiographic data for 25 patients who underwent mechanical thrombectomy with the Solitaire stent for acute BAO were identified from this data base between December 2010 and December 2012. During this period, a total of 133 consecutive patients with acute ischemic stroke caused by intracranial large-artery occlusion were treated with mechanical thrombectomy by use of the Solitaire stent as first-line endovascular therapy. The institutional ethics committee approved this retrospective analysis and waived informed consent on the basis of study design.

On admission, all patients were evaluated by a stroke neurologist and assessed by means of the NIHSS. All patients underwent an initial imaging protocol that included non-enhanced CT scan and multimodal MR imaging. The inclusion criteria for endovascular treatment were as follows: 1 ) baseline NIHSS score $\geq 4 ; 2$ ) no intracerebral hemorrhage detected on the cranial CT or MR imaging; 3) BAO detected with $\mathrm{MR}$ angiography and conventional angiography; 4) no bilateral diffuse pontine ischemia on the DWI; and 5) the start of the procedure within 8 hours after symptom onset. IV rtPA $(0.9 \mathrm{mg} / \mathrm{kg})$ was administered in patients presenting within 4.5 hours from symptom onset. Subsequent endovascular treatment as a rescue therapy was considered within 1 hour of IV rtPA in patients with no neurologic improvement, defined as a NIHSS score unchanged from baseline or a worsening neurologic deficit.

\section{Endovascular Treatment}

All endovascular therapy was performed by 1 interventional neuroradiologist with 10 years of experience in neurovascular intervention. Written informed consent for endovascular therapy was obtained from a family member of all patients. Cerebral angiography and endovascular therapy were performed by means of a femoral approach under conscious sedation. In cases of agitation, an IV bolus of midazolam was given and repeated if necessary. After an arterial occlusion was demonstrated with diagnostic angiography, a diagnostic catheter was exchanged for a $6 \mathrm{~F}$ or $7 \mathrm{~F}$ guide catheter, which was placed in the most accessible or dominant vertebral artery. A balloon guide catheter was not used in any patient.

In all patients, mechanical thrombectomy with a Solitaire stent, which was $4 \mathrm{~mm}$ in diameter and $20 \mathrm{~mm}$ long, was used as a first-line endovascular treatment method. A microcatheter with a 0.021 -inch internal diameter was navigated distal to the clot over a 0.014 -inch microwire. The Solitaire stent was then introduced through the microcatheter and fully deployed across the occluded segment. After the stent was maintained in place for
1-3 minutes, the fully deployed stent and the delivery microcatheter were slowly pulled back together and withdrawn outside the body through the guide catheter. During clot retrieval, continuous manual aspiration with a $50-\mathrm{mL}$ syringe at the guide catheter was performed. After removal of the Solitaire stent and microcatheter, another $10 \mathrm{~mL}$ of blood was aspirated from the guide catheter to prevent re-embolization of a vagrant clot. A control angiogram was performed to assess the recanalization status and possible distal embolic events. If recanalization was unsuccessful, the procedure was repeated. A maximum of 5 retrieval attempts was allowed.

When Solitaire thrombectomy was unsuccessful, additional rescue endovascular procedures were performed, including forced suction thrombectomy by use of a reperfusion catheter (Penumbra, Alameda, California) or aggressive mechanical clot disruption and IA urokinase infusion. The details of the technique for aggressive mechanical clot disruption have been described previously. ${ }^{16}$ If an underlying atherosclerotic stenosis was revealed during the procedure, balloon angioplasty with or without stent placement was performed after the Solitaire thrombectomy.

During the procedure, heparin or glycoprotein IIb/IIIa inhibitor was not administered-IV or IA-in any patient. Patients who underwent intracranial angioplasty with or without stent placement received aspirin and clopidogrel (Plavix) for at least 3 months after the procedure. After the procedure, patients were admitted to an intensive care unit. All patients underwent nonenhanced CT scans immediately following and 24 hours after endovascular therapy. The start of endovascular therapy was defined as the moment the needle punctured the common femoral artery. Recanalization status was assessed on the final angiogram and classified according to the modified TICI scale. ${ }^{17}$ Successful recanalization was defined as a TICI grade of $2 \mathrm{~b}$ or 3 . Assessment of angiographic images was performed by the consensus of 2 experienced neuroradiologists who were blinded to the procedure.

\section{Outcome Measures}

For all patients, we analyzed medical records to determine age, sex, risk factors, stroke subtype according to the Trial of Org 10172 in Acute Stroke Treatment classification, baseline NIHSS score, use of IV rtPA, time to IV rtPA and endovascular therapy, duration of the procedure, presence or absence of symptomatic hemorrhage, recanalization status, procedure-related vessel perforation and dissection, NIHSS score at discharge, and clinical outcome. Symptomatic hemorrhage was defined as a parenchymal hematoma that caused a mass effect on CT scans, with clinical deterioration defined as a $\geq 4$-point increase in the NIHSS score or a 1-point deterioration in the level of consciousness. Vessel perforation was defined as obvious angiographic contrast extravasation that occurred during the procedure. Arterial dissection was defined as an identifiable intimal flap on the control angiogram obtained after mechanical thrombectomy. Neurologic evaluation was performed immediately by a stroke neurologist, 24 hours and 3 months after treatment, when any change occurred in clinical symptoms, and before the patient was discharged. Clinical outcome was assessed by means of the mRS by a stroke neurologist 3 months after treatment. Good clinical outcome was defined as an $\mathrm{mRS}$ score $\leq 2$. 


\begin{tabular}{|c|c|c|c|c|}
\hline & Good Outcome ( $n=12$ ) & Poor Outcome $(n=13)$ & Total $(n=25)$ & $P$ \\
\hline Age, y (mean $\pm S D)$ & $63.2 \pm 16.86$ & $71.8 \pm 11.92$ & 68 & NS \\
\hline Sex, male, $n(\%)$ & $6(50 \%)$ & $8(61.5 \%)$ & $14(56 \%)$ & NS \\
\hline \multicolumn{5}{|l|}{ Risk factors } \\
\hline Hypertension & $3(25 \%)$ & $12(92.3 \%)$ & $15(60 \%)$ & .001 \\
\hline Atrial fibrillation & $5(41.7 \%)$ & $4(30.8 \%)$ & $9(36 \%)$ & NS \\
\hline Diabetes mellitus & $4(33.3 \%)$ & $4(30.8 \%)$ & $8(32 \%)$ & NS \\
\hline Dyslipidemia & $4(33.3 \%)$ & $2(15.4 \%)$ & $6(24 \%)$ & NS \\
\hline Smoking & $3(25 \%)$ & $3(23.1 \%)$ & $6(24 \%)$ & NS \\
\hline History of stroke or TIA & $0 \%$ & $4(30.8 \%)$ & $4(16 \%)$ & NS \\
\hline Coronary artery disease & $0 \%$ & $2(15.4 \%)$ & $2(8 \%)$ & NS \\
\hline Patent foramen ovale & $1(8.3 \%)$ & $0 \%$ & $1(4 \%)$ & NS \\
\hline Valvular heart disease & $1(8.3 \%)$ & $0 \%$ & $1(4 \%)$ & NS \\
\hline Atrioventricular block & $0 \%$ & $1(8.3 \%)$ & $1(4 \%)$ & NS \\
\hline Intravenous thrombolysis & $3(25 \%)$ & $3(23.1 \%)$ & $6(24 \%)$ & NS \\
\hline Time to procedure, min & $260 \pm 100.32$ & $290 \pm 74.42$ & $285 \pm 88.48$ & NS \\
\hline Procedure time, $\min$ & $27.5 \pm 24.21$ & $30 \pm 20.35$ & $30 \pm 21.91$ & NS \\
\hline Time to recanalization, min & $300 \pm 110.03$ & $310 \pm 91.23$ & $310 \pm 99.91$ & NS \\
\hline \multicolumn{5}{|l|}{ Rescue treatment } \\
\hline Clot disruption with intra-arterial urokinase & $1(8.3 \%)$ & $0 \%$ & $1(4 \%)$ & NS \\
\hline Angioplasty with or without stenting & $3(25 \%)$ & $3(23.1 \%)$ & $6(24 \%)$ & NS \\
\hline Baseline NIHSS score & $9.5 \pm 3.13$ & $14 \pm 5.75$ & 11 & .005 \\
\hline Discharge NIHSS score & $2 \pm 2.57$ & $9 \pm 8.21$ & 4 & .003 \\
\hline \multicolumn{5}{|l|}{ Stroke etiology } \\
\hline Large-artery atherosclerosis & $4(33.3 \%)$ & $5(38.5 \%)$ & $9(36 \%)$ & NS \\
\hline Cardioembolic & $6(50 \%)$ & $6(46.2 \%)$ & $12(48 \%)$ & NS \\
\hline Undetermined & $2(8.3 \%)$ & $2(15.4 \%)$ & $4(16 \%)$ & NS \\
\hline
\end{tabular}

Note:-NS indicates non-significant.

\section{Statistical Analysis}

Statistical analyses were performed with the use of SPSS software (Version 19.0; IBM, Armonk, New York). The relationship between the characteristics and 3-month clinical outcome was determined by bivariate analysis. The $\chi^{2}$ test was used for categoric variables and the Mann-Whitney $U$ test for continuous variables. A value of $P<.05$ was considered significant.

\section{RESULTS}

Data from 25 patients (14 men and 11 women) were analyzed. Baseline patient characteristics according to clinical outcome are shown in the Table. Twenty-three patients had BAO only, and 2 patients had long segment occlusion from the distal intracranial segment of the vertebral artery to the basilar artery.

The median NIHSS score on admission was 11, with scores ranging from 3-25. The median time from symptom onset to endovascular therapy was 285 minutes (range, 110-470 minutes), the median procedure time was 30 minutes (range, 13-100 minutes), and the median time to recanalization was 310 minutes (range, 132-560). IV rtPA was administered in 6 patients (24\%) before mechanical thrombectomy.

Successful recanalization was achieved in 96\% (24/25) of patients, and complete recanalization (TICI grade 3 ) occurred in $76 \%(19 / 25)$ of patients. Successful recanalization with the Solitaire thrombectomy alone was achieved in $84 \%(21 / 25)$ of patients. In this study, rescue treatment was needed in 4 patients after Solitaire thrombectomy failed. One patient (patient 1) received aggressive mechanical clot disruption and low-dose IA urokinase infusion after unsuccessful Solitaire thrombectomy. Urokinase (80,000 IU) was given as a single bolus injection in this patient. Three patients (patients 10, 20, and 23) received forced suction thrombectomy with the Penumbra reperfusion catheter.
Additional successful recanalization occurred in 3 of 4 patients who underwent rescue treatments. Three patients who underwent rescue forced suction thrombectomy with Penumbra reperfusion catheter achieved complete recanalization (TICI grade 3 ). One patient failed to achieve successful recanalization after aggressive mechanical clot disruption and IA urokinase infusion, and the patient's final recanalization grade was TICI $2 \mathrm{a}$.

Six patients (24\%) had underlying atherosclerotic stenosis in the distal intracranial vertebral artery $(n=3)$, basilar artery $(n=$ $2)$, or both the distal vertebral and basilar arteries $(n=1)$. In these 6 patients, angioplasty, with or without stent placement, was performed to treat underlying stenosis after successful recanalization with a Solitaire thrombectomy. Of these 6 patients, 4 patients received angioplasty with stent placement and 2 patients received angioplasty alone. No angioplasty-related complication occurred in these 6 patients.

No patient had symptomatic hemorrhage during their hospital stay. At discharge, the NIHSS score was improved (decrease $\geq 4$ points) in 15 patients (60\%; range, $0-24$; median, 4 ). At the 3-month follow-up, 12 patients (48\%) showed a good clinical outcome. Eighty percent (12/15) of patients with hypertension had a poor clinical outcome and 90\% (9/10) of those without hypertension had a good outcome; this difference was statistically significant $(P=.001)$. Median NIHSS scores on admission $(9.5$ versus $14, P=.005$ ) and at discharge (2 versus $9, P=.003$ ) were significantly lower in patients with a good outcome than in those with a poor outcome. Other variables including age, sex, risk factors other than hypertension, stroke subtype, time to treatment, and procedure time were not statistically associated with a good clinical outcome. There was a good outcome in 50\% (3/6) of patients who received a Solitaire thrombectomy after failed IV 
thrombolysis and in 47\% (9/19) of those who received a Solitaire thrombectomy as an initial treatment $(P=.910)$. The median time from symptom onset to endovascular therapy tended to be shorter in patients with a good clinical outcome than in those with a poor clinical outcome (270 versus 313 minutes, $P=.301$ ).

Procedure-related complications occurred in 2 patients ( $8 \%)$. There was 1 vessel rupture unrelated to the Solitaire device. Active contrast extravasation caused by perforation of the thalamoperforating artery by a microwire was observed in 1 patient (patient 16). This patient exhibited a localized hematoma in the left paramedian thalamus, which was resolved on follow-up CT and had no neurologic worsening. One patient (patient 14) had SAH on the immediate posttherapeutic CT scan. This patient exhibited no postprocedural neurologic deterioration or associated symptomatic parenchymal hemorrhage. There were no device-related complications. The mortality rate was $12 \%(3 / 25)$ at 3 months. Two patients died 10 and 18 days after stroke onset because of extensive brain stem infarction. One patient with mRS score of 5 at discharge died 90 days after an initial stroke because of pneumonia.

\section{DISCUSSION}

The present study demonstrates that mechanical thrombectomy by use of the Solitaire stent within 8 hours of stroke onset in acute $\mathrm{BAO}$ is associated with increased rates of both good outcome and survival compared with previous studies. In our study, 88\% (22/ 25 ) of patients survived for 3 months, and more than half of the survivors $(55 \%, 12 / 22)$ had a good outcome.

There are still only a few studies that have investigated the clinical results of mechanical thrombectomy with stent retrievers in patients with acute BAO. ${ }^{12-14}$ In contrast to our study, these previous studies included patients treated within 24 hours of stroke symptom onset. In a series of 14 patients treated with a Solitaire thrombectomy, Mordasini et $\mathrm{al}^{12}$ reported that $28.6 \%$ $(4 / 14)$ had a good outcome (mRS $0-2)$, and the mortality rate was $35.7 \%(5 / 14)$. In their study, a Solitaire thrombectomy was not the first-line endovascular treatment, and the median time from symptom onset to first angiogram was 414 minutes (range, 1761440 minutes). Espinosa et $\mathrm{al}^{13}$ reported a series of 18 patients with acute $\mathrm{BAO}$ who were directly treated with mechanical thrombectomy with either the Solitaire stent $(n=10)$ or the Trevo stent $(n=8)$. In their study, good functional outcome (mRS $0-2)$ was achieved in 50\% (9/18), the mortality rate was $22.3 \%$ at 3 months, and time from symptom onset to groin puncture was 366 minutes. Most recently, in a series of 31 patients treated with Solitaire thrombectomy as the first-line endovascular treatment, Mourand et $\mathrm{al}^{14}$ reported that 35\% (11/31) had a good outcome, the mortality rate was $32 \%$ (10/31), and mean time from symptom onset to recanalization was 512 minutes. This group did not provide data regarding the time to treatment.

In comparison with these previous studies, the good outcome rate in our study is among the highest, and the mortality rate is among the lowest. In our study, the median time to treatment was $<5$ hours (285 minutes) and the median time to recanalization was $<6$ hours ( 310 minutes). The shorter time window for mechanical thrombectomy could be one of the factors that contributed to the good clinical results obtained in our study. A recent post hoc analysis of the BASICS trial found that early recanalization therapy in patients with $\mathrm{BAO}$ was associated with a more favorable outcome with a significantly increased chance of a poor outcome when recanalization therapy was started $>6$ hours after symptom onset. ${ }^{15}$ In the BASICS trial, $85 \%$ of patients who were treated $>9$ hours after stroke onset had a poor functional outcome, whereas only $62 \%$ of those treated at $\leq 3$ hours had a poor outcome. ${ }^{15}$ The results of our study suggest that the same situation also holds true for mechanical thrombectomy with stent retrievers in patients with acute BAO.

Several other prognostic factors have been described that predict a good clinical outcome in patients with acute BAO receiving recanalization therapy and include recanalization status, baseline NIHSS score, and the extent of ischemic changes on the pretreatment imaging studies. ${ }^{18-22}$

A systematic review including 316 patients in 10 IA thrombolysis studies found that recanalization was associated with a substantial reduction in mortality rates in patients with acute BAO (mortality rate of $87 \%$ in non-recanalized compared with $39 \%$ in recanalized patients; $P<.001) .{ }^{18}$ The overall recanalization rate of these 10 studies was $64 \%$, with a $56 \%$ mortality rate. Recent studies with the use of mechanical thrombectomy with stent retrievers reported significantly increased recanalization rates ranging from $74-100 \%$ in patients with acute BAO. ${ }^{12-14}$ In our study, we achieved successful recanalization (TICI grades $2 \mathrm{~b}$ to 3 ) in $96 \%(24 / 25)$ of patients, which is in line with previous reports. Successful recanalization with only the Solitaire stent occurred in $84 \%(21 / 25)$ of patients. This high rate of recanalization might also have contributed to the good results obtained in our study.

In a series of 106 patients with BAO treated with IA thrombolysis from 1992-2010, Jung et $\mathrm{al}^{19}$ found that lower NIHSS score on admission was an independent predictor of good or moderate clinical outcome (mRS $0-3)(P<.0001)$ and survival $(P=.012)$ at 3 months. The results of our study are in line with Jung's study. In our study, the median NIHSS score at admission was significantly lower in patients with a good outcome (mRS 0-2) than in those with a poor outcome (9.5 versus $14, P=.005)$. With regard to baseline NIHSS score, the median NIHSS score on admission in our study population was 11 , which was lower than those of previous studies. Lower baseline NIHSS score could also be one of the factors that contributed to the good results obtained in our study.

The extent of early ischemic changes on pretreatment imaging studies can be used as a prognostic factor of clinical outcome in patients treated with recanalization therapy. ${ }^{20-22}$ Puetz et $\mathrm{al}^{20}$ showed that the posterior circulation ASPECTS on CT angiography source images predicts good outcome or death within 1 month in the subgroup of the BASICS registry population. Of 158 patients, $23 \%(18 / 78)$ of patients with a posterior circulation ASPECTS $\geq 8$ had a good outcome, whereas $11 \%$ (9/80) of those with a posterior circulation ASPECTS $<8$ had a good outcome. The mortality rate was $32 \%(25 / 78)$ in patients with a posterior circulation ASPECTS $\geq 8$ and $55 \%$ (44/80) in patients with a posterior circulation ASPECTS $<8$. We did not assess the extent of early ischemic changes on pretreatment CT or MR imaging in this study.

In our study, rescue treatment was needed in 4 patients (forced 
suction thrombectomy in 3 and mechanical clot disruption with IA urokinase in 1) after unsuccessful Solitaire thrombectomy. Of these 4 patients, subsequent successful recanalization after a rescue treatment occurred in 3 patients who underwent forced suction thrombectomy with the Penumbra catheter, and 2 of these 4 patients showed a good outcome at 3 months. Therefore, adding suction thrombectomy with the Penumbra device appears to be promising in cases of unsuccessful thrombectomy with the Solitaire stent in patients with acute BAO. The effectiveness of such treatment must be confirmed in future studies.

Our study confirms the safety of mechanical thrombectomy with the Solitaire stent in patients with BAO. There was no symptomatic hemorrhage after mechanical thrombectomy in this study, and no vessel rupture or arterial dissections related to the Solitaire device occurred. There was 1 rupture of a perforating artery because of microwire perforation and $1 \mathrm{SAH}$, neither of which caused postprocedural neurologic deterioration. A recent study suggested that SAH might occur after mechanical thrombectomy with the Solitaire stent because of angiographically occult ruptures of small vessels caused by mechanical stretch during stent retrieval and that this had a benign clinical course. ${ }^{23}$ Mordasini et $\mathrm{al}^{12}$ reported no device-related complications or symptomatic hemorrhage. Espinosa de Rueda et $\mathrm{al}^{13}$ reported $1 \mathrm{SAH}$ in 18 patients with BAO treated with primary mechanical thrombectomy with the Solitaire stent. They reported a symptomatic hemorrhage rate of $0 \%$, similar to that in our study. In a series of 31 patients with BAO treated with a Solitaire thrombectomy, Mourand et $\mathrm{al}^{14}$ reported that vertebral artery dissection occurred in 1 patient, and no vessel perforation was observed. They also reported that symptomatic hemorrhage occurred in $16 \%(5 / 31)$ of patients, which is quite frequent compared with other reports. They did not describe the use of rescue treatments, including IA thrombolysis, in their report.

The limitations of our study included the small number of patients and the lack of a control group.

\section{CONCLUSIONS}

The current study suggests that a good clinical outcome can be safely achieved in nearly half of patients with acute BAO by use of mechanical thrombectomy with the Solitaire stent if treatment is started within 8 hours of symptom onset. In addition, such treatment can significantly reduce the mortality rate in this subset of patients with acute stroke, who otherwise have an extremely poor prognosis.

\section{REFERENCES}

1. Hacke W, Zeumer H, Ferbert A, et al. Intra-arterial thrombolytic therapy improves outcome in patients with acute vertebrobasilar occlusive disease. Stroke 1988;19:1216-22

2. Schonewille WJ, Wijman CA, Michel P, et al. Treatment and outcomes of acute basilar artery occlusion in the basilar artery international cooperation study (BASICS): a prospective registry study. Lancet Neurol 2009;8:724-30

3. Lindsberg PJ, Mattle HP. Therapy of basilar artery occlusion: a systematic analysis comparing intra-arterial and intravenous thrombolysis. Stroke 2006;37:922-28

4. Saver JL, Jahan R, Levy EI, et al. Solitaire flow restoration device versus the Merci retriever in patients with acute ischaemic stroke
(SWIFT): a randomized, parallel-group, non-inferiority trial. Lancet 2012;380:1241-49

5. Nogueiro RG, Lutsep HL, Jovin TG, Aet al. Trevo versus Merci retrievers for thrombectomy revascularization of large vessel occlusions in acute ischemic stroke (TREVO 2): a randomized trial. Lancet 2012;380:1231-40

6. Castano C, Dorado L, Guerrero C, et al. Mechanical thrombectomy with the Solitaire $\mathrm{AB}$ device in large artery occlusions of the anterior circulation: a pilot study. Stroke 2010;41:1836-40

7. Roth C, Papanagiotou P, Behnke S, et al. Stent-assisted mechanical recanalization for treatment of acute intracerebral artery occlusions. Stroke 2010;41:2559-67

8. Costalat V, Machi P, Lobotesis K, et al. Rescue, combined, and standalone thrombectomy in the management of large vessel occlusion stroke using the Solitaire device: a prospective 50-patient singlecenter study: timing, safety, and efficacy. Stroke 2011;42:1929-35

9. Machi P, Costalat V, Lobotesis K, et al. SolitaireFR thrombectomy system: immediate results in $\mathbf{5 6}$ consecutive acute ischemic stroke patients. J Neurointerv Surg 2012;4:62-66

10. Miteff F, Faulder KC, Goh AC, et al. Mechanical thrombectomy with a self-expanding retrievable intracranial stent (Solitaire $A B$ ): experience in 26 patients with acute cerebral artery occlusion. AJNR Am J Neuroradiol 2011;32:1078-81

11. Yoon YH, Yoon W, Jung MY, et al. Outcome of mechanical thrombectomy with Solitaire stent as first-line intra-arterial treatment in intracranial internal carotid artery occlusion. Neuroradiology 2013; 55:999-1005

12. Mordasini P, Brekenfeld C, Byrne JV, et al. Technical feasibility and application of mechanical thrombectomy with the Solitaire FR revascularization device in acute basilar artery occlusion. AJNR Am J Neuroradiol 2013;34:159-63

13. Espinosa de Rueda M, Parrilla G, Zamarro J, et al. Treatment of acute vertebrobasilar occlusion using thrombectomy with stent retrievers: initial experience with 18 patients. AJNR Am J Neuroradiol 2013;34:1044-48

14. Mourand I, Machi P, Milhaud D, et al. Mechanical thrombectomy with the Solitaire device in acute basilar artery occlusion. J Neurointervent Surg 2014;6:200-04

15. Vergouwen MD, Algra A, Pfefferkorn T, et al. Time is brain(stem) in basilar artery occlusion. Stroke 2012;43:3003-06

16. Yoon W, Park MS, Cho KH. Low-dose intra-arterial urokinase and aggressive mechanical clot disruption for acute ischemic stroke after failure of intravenous thrombolysis. AJNR Am J Neuroradiol 2010;31:161-64

17. Higashida RT, Furlan AJ, Roberts $\mathrm{H}$, et al. Trial design and reporting standards for intra-arterial cerebral thrombolysis for acute ischemic stroke. Stroke 2003;34:e109-37

18. Smith WS. Intra-arterial thrombolytic therapy for acute basilar artery occlusion: pro. Stroke. 2007;38:701-03

19. Jung S, Mono ML, Fischer U, et al. Three-month and long-term outcomes and their predictors in acute basilar artery occlusion treated with intra-arterial thrombolysis. Stroke 2011;42:1946-51

20. Puetz V, Khomenko A, Hill MD, et al. Extent of hypoattenuation on CT angiography source images in basilar artery occlusion: prognostic value in the Basilar Artery International Cooperation Study. Stroke 2011;42:3454-59

21. Tei H, Uchiyama S, Usui T, et al. Posterior circulation ASPECTS on diffusion-weighted MRI can be a powerful marker for predicting functional outcome. J Neurol 2010;257:767-73

22. Strbian D, Sairanen T, Silvennoinen $H$, et al. Thrombolysis of basilar artery occlusion: impact of baseline ischemia and time. Ann Neurol 2013;73:688-94

23. Yoon W, Jung MY, Jung SH, et al. Subarachnoid hemorrhage in a multimodal approach heavily weighted toward mechanical thrombectomy with Solitaire stent in acute stroke. Stroke 2013; 44:414-19 